\title{
Comparison of adapalene $0.1 \%$ solution and tretinoin $0.025 \%$ gel in the topical treatment of acne vulgaris
}

\author{
C.N.ELLIS, L.E.MILLIKAN,* E.B.SMITH,† D.M.CHALKER, + L.J.SWINYER,§ \\ I.H.KATZ, $\uparrow$ R.S.BERGER, ${ }^{*}$ O.H.MILLS JR, ${ }^{* *}$ M.BAKER, $\dagger \dagger$ M.VERSCHOORE†† AND \\ C.LOESCHE $\S$ \\ Department of Dermatology, University of Michigan Medical School, Ann Arbor, MI, *Department of Dermatology, Tulane University \\ Medical Center, New Orleans, LA, †Department of Dermatology, University of Texas Medical Branch, Galveston, TX, $\$$ Department of \\ Dermatology, Medical College of Georgia, August, GA, §Private Practice, Salt Lake City, UT, $\uparrow$ Private Practice, Fridley, MN, **Hill \\ Top Research, UMDNJ, Robert Wood Johnson Medical School, East Brunswick, NJ, ††Galderma Laboratories, Inc., Fort Worth, TX, \\ HtLaboratoires Galderma, Levallois-Perret, France, §§Galderma R\&D, Sophia Antipolis, France
}

Summary A multicentre study was conducted to compare clinical safety and efficacy of adapalene $0 \cdot 1 \%$ solution and tretinoin $0.025 \%$ gel, both topical treatments for acne, in a once-daily dosage regimen for 12 weeks. A total of 297 patients were enrolled by eight investigators in this randomized, investigator-masked study in a parallel group design. An open label period using adapalene followed this study to assess the long-term safety of adapalene solution.

Adapalene and tretinoin proved to be clinically and statistically effective in treating acne by reducing inflammatory ( $47 \%$ and $50 \%$, respectively) and non-inflammatory lesions ( $57 \%$ and $54 \%$ ) as compared to baseline. When comparing patients who had $75 \%$ or greater improvement in open comedones, adapalene was shown to be significantly more effective than tretinoin. No serious adverse event was reported during this study, including during the long-term period. The reactions that occurred were similar between treatments, i.e. burning, pruritus, scaling, dryness and erythema.

Adapalene, a novel topical retinoid receptor agonist has been developed for the treatment of acne vulgaris by CIRD Galderma in France. It has been commercialised in Europe since 1995 as an aqueous gel (Differin ${ }^{\circledR}$ gel) and has shown good efficacy and tolerance in the topical treatment of acne vulgaris. ${ }^{1-4}$ The profile of adapalene as a molecule is that of a retinoid, since it acts as a retinoid agonist, but the affinity profile for nuclear and cytosolic retinoic acid receptors is different from that of tretinoin. ${ }^{5}$ Whereas tretinoin binds to all RARs $(\alpha$, $\beta$ and $\gamma$ ) adapalene has selective affinity to RAR- $\beta$ and RAR- $\gamma$. Additionally, adapalene is very stable even in presence of a strong oxidizer and light and is highly lipophilic. ${ }^{5}$ After application of an aqueous gel formulation, it shows high concentrations in the follicle ${ }^{6}$ (Fig. 1).

In clinical practice up to now, selection of the retinoid formulation is directly related to cutaneous adverse effects. Creams seem to be well tolerated, gels (alcoholic) and solutions tend to cause more irritation. Ideally, a non-greasy formulation, a gel or solution should provide the best balance of cosmetic acceptability and tolerance.
This study was conducted to compare efficacy and safety of adapalene $0 \cdot 1 \%$ solution in a large number of patients with acne vulgaris, with those of a marketed product, tretinoin $0 \cdot 025 \%$ gel.

\section{Materials and methods}

This multicentre study was designed to evaluate and compare the safety and efficacy of adapalene $0.1 \%$ solution (Differin ${ }^{\circledR} 0 \cdot 1 \%$ solution, Galderma Lab) with tretinoin $0.025 \%$ gel (Retin A ${ }^{\circledR} 0.025 \%$ gel, Johnson \& Johnson) in patients with acne vulgaris. Two hundred and ninety-seven patients (149 receiving adapalene and 148 tretinoin) were enrolled by eight investigators in this randomized, investigator-masked, multicentre, parallel group comparison. All consentaged patients signed an IRB (Institutional Review Board) approved written informed consent form. Patients below the age of consent signed the consent form as did their parent(s) or guardian(s). At week 12, at five sites patients from both groups were given the option to continue under open label conditions with adapalene 


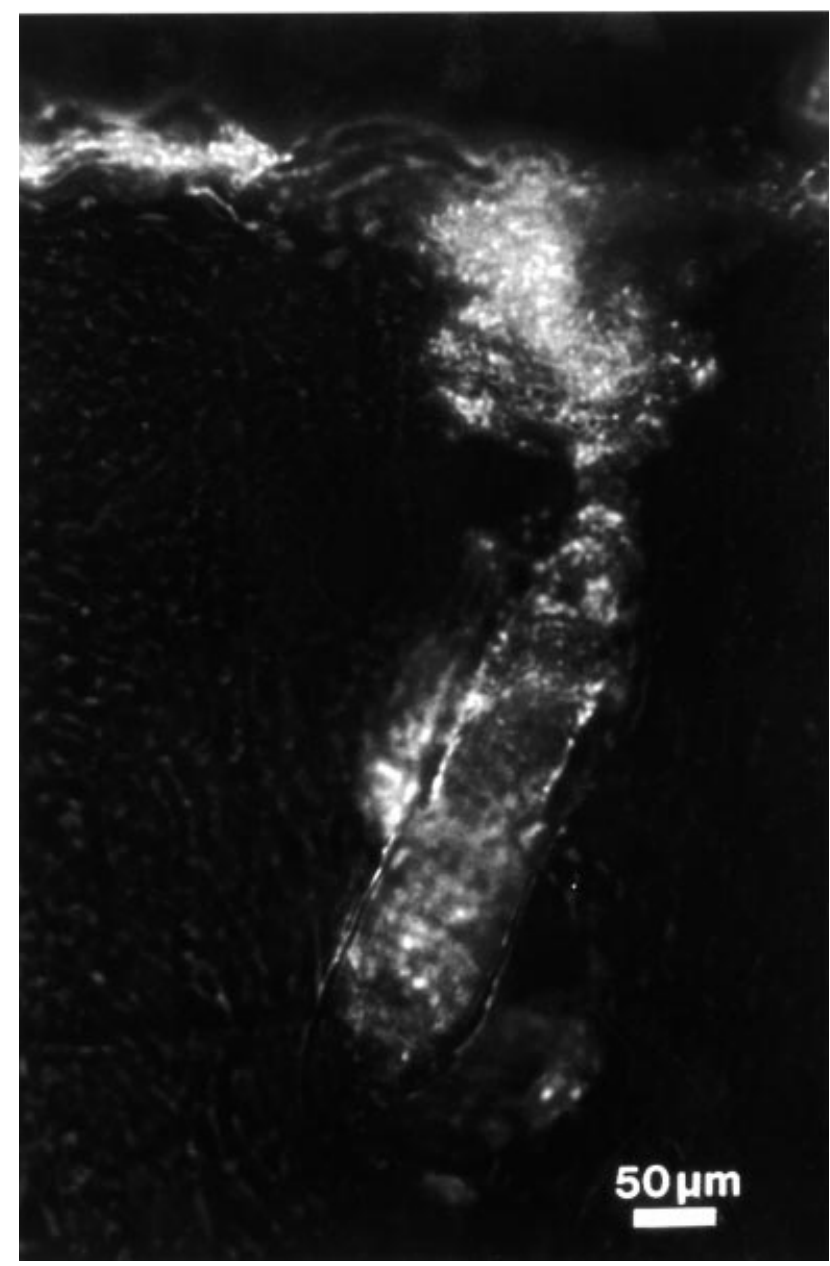

Figure 1. Skin distribution of adapalene microcrystals 5 min after in vitro topical application to human skin (fluorescence microscopy).

$0 \cdot 1 \%$ solution for 9 additional months to assess longterm safety with visits every three months.

Patients met the following inclusion and exclusion criteria: male and female patients aged between 12 and 30 years inclusive, presenting at least 20 noninflammatory lesions and 10 inflammatory lesions on the face with a global assessment of grade 1-5 inclusive, based on the acne severity using representative photographs. ${ }^{7}$ Female patients using birth control pills had to have used them for at least 3 months before inclusion into the study, and were to continue during and for one month after the end of the study. A pregnancy test was performed prior to admission, at each follow-up visit and one month after the last dose of study medication. Patients with secondary acne, severe acne (acne fulminans, acne conglobata) were not included. Wash-out periods for oral isotretinoin (six months), oral antibiotics except penicillin or systemic anti-inflammatory drugs (four weeks) and for topical acne treatment (two weeks) had to be respected. Any prolonged use of interfering therapy was an exclusion criteria.

Patients were instructed to apply the study products to the entire face once daily at bedtime for 12 weeks. Dosage regimen could be altered occasionally to up to two weeks to accommodate a patient who complained of skin discomfort. The study products could be applied to chest and/or back in the case of acne involvement of those areas. Efficacy was evaluated by analysing changes in the numbers of facial noninflammatory lesions (open and closed comedones) and inflammatory lesions (papules, pustules, nodules and cysts). Evaluation was made at baseline, weeks 2, 4,8 and 12 (and for the extension period at months 6 , 9 and 12). Lesions were counted on the face above the jawline, excluding the nose at each timepoint; global assessment was also conducted using the Cunliffe scale. ${ }^{7}$ At each patient's final visit the investigator was asked to decide whether or not the patient's acne condition had improved.

Facial skin comfort was assessed by scoring erythema, dryness, oiliness, scaling, burning and pruritus on a four point scale - from absent to severe. The latter two parameters were assessed as a chronic and as an immediate response following drug application. Adverse events and concomitant therapy were recorded during the trial. In two centres, blood (standard chemistries, haematologies) and urine samples were obtained at baseline and at week 12 .

Efficacy parameters were analysed at all time-points of the 12-week trial including endpoint analysis (last available treatment visit for a given patient in the intent-to-treat analysis; the last observation being carried forward). Cochran-Mantel-Haenszel test of rank scores for global assessment, safety parameters and percentage changes of lesion counts controlling for investigator differences was used. Lesion counts of each individual type were analysed by a non-parametric median test. Analysis of covariance was performed on square-root transformed counts. All statistical analyses were performed using a two-sided test with 0.05 significance level.

\section{Results}

\section{Study population}

Two hundred and ninety-seven patients (152 males and 145 females) were enrolled with 149 assigned to adapalene $0 \cdot 1 \%$ solution and 146 to tretinoin $0 \cdot 025 \%$ 
gel. Each centre enrolled about 40 patients. No significant differences between groups were observed in the study population with regard to age, sex, race and concomitant therapy at baseline. The age range in the adapalene group was $12-30$ years with a mean age of 19. For tretinoin, the range was $12-32$ years with a mean age of 18. Data of two patients entered in the study at age 32 were included in the analysis. Two hundred and fifty-nine patients were considered evaluable for efficacy, 38 were excluded from the perprotocol analysis because they had no or less than 4 weeks follow-up. Thirty-five patients in the adapalene group and 22 with tretinoin discontinued early (before 12 weeks) for various reasons (lost-to follow-up, patient's request, non-compliance and interfering therapy or interfering conditions). Twenty-five patients using adapalene and 20 using tretinoin altered the dosage regimen at some time. Seventy-two patients entered the extension period of up to nine additional months; 59 of those were considered evaluable.

\section{Efficacy}

For both treatments, primary criteria (total lesion count, non-inflammatory lesion and inflammatory lesion counts) showed significant reductions from baseline to week 12 . The mean percentage reduction compared to baseline for non-inflammatory lesions was $57 \%$ ( $55 \%$ at endpoint) for adapalene and $54 \%$ ( $52 \%$ at endpoint) for tretinoin. Inflammatory lesions were reduced by $47 \%$ ( $46 \%$ at endpoint) in the adapalene group and $50 \%(45 \%$ at endpoint $)$ in the tretinoin group. Analysis of total lesions showed a reduction of $54 \%$ at week 12 and $52 \%$ at endpoint for adapalene and $52 \%$ and $49 \%$, respectively, for tretinoin (Table 1). The lesion counts are shown in Figs 2-4. Despite the numerical differences, no statistically significant differences between treatments were detected. The percentage reduction in lesions was analysed utilizing four categories of improvement (excellent, good, fair and poor). A significant difference in the reduction of open comedones between treatments in the 'excellent improvement' category was observed in favour of

Table 1 Mean percentage reduction in non-inflammatory and inflammatory lesions at 12 weeks compared to baseline

\begin{tabular}{lcccc}
\hline & $\begin{array}{c}\text { Non- } \\
\text { Week 12 }\end{array}$ & $\begin{array}{c}\text { inflammatory } \\
\text { Week 12 }\end{array}$ & $\begin{array}{c}\text { Inflammatory } \\
\text { Week 12 }\end{array}$ & $\begin{array}{c}\text { Total } \\
\text { Week 12 }\end{array}$ \\
\hline Adapalene & 111 & 57 & 47 & 54 \\
Tretinoin & 126 & 54 & 50 & 52 \\
\hline
\end{tabular}

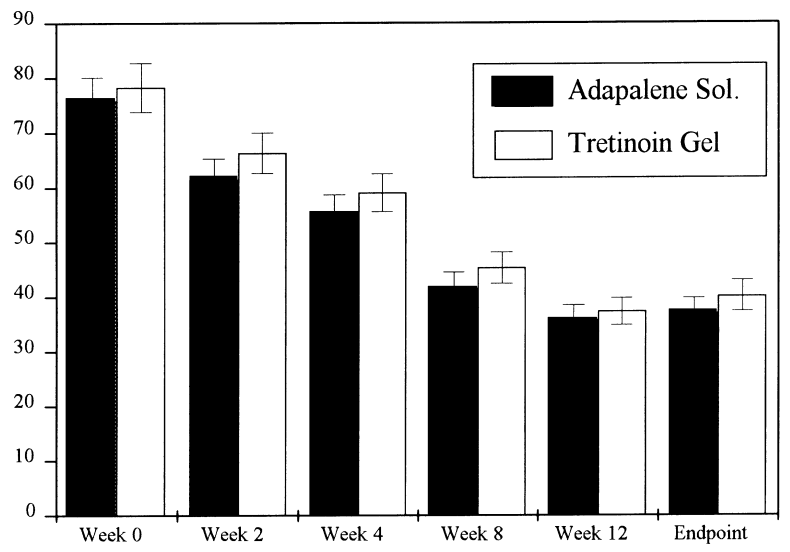

Figure 2. Total lesion count.

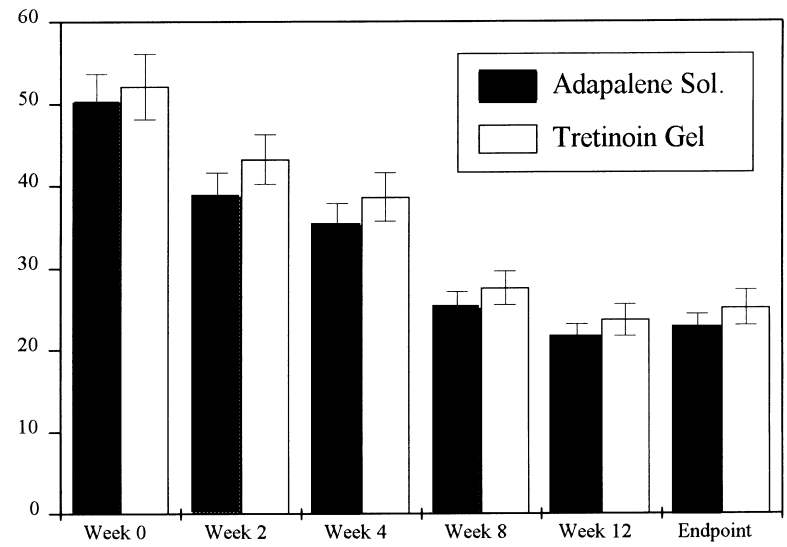

Figure 3. Non-inflammatory lesion count.

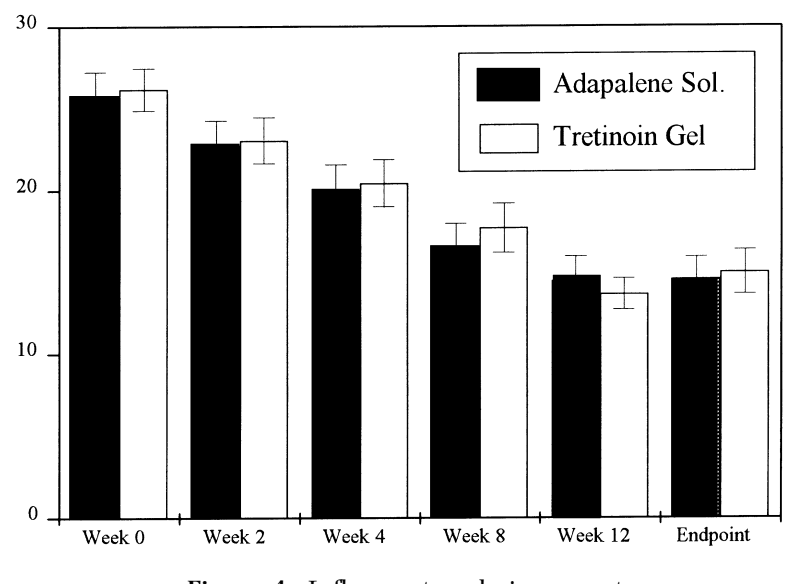

Figure 4. Inflammatory lesion count 
adapalene (Fig. 5); however, a separate statistical analysis of acne lesions did not detect any differences between treatments for open and closed comedones, papules, pustules, nodules and cysts.

Global assessment based on the acne severity grade ${ }^{7}$ demonstrated that both treatments were equally effective, showing significant improvement at each evaluation point $(P<0.001)$, but no significant differences between treatments. Patients treated with adapalene improved from 1.52 at baseline to 0.93 at week 12; the data for tretinoin treated patients was very comparable (1.53 at baseline, 0.94 at week 12 ). The physician's opinion of patient's improvement at the final visit again revealed no differences between treatments: $88.4 \%$ of those using adapalene and $89.6 \%$ of the tretinoin group were judged to be improved.

Since safety was the primary criterion during the long-term extension, only efficacy data concerning total lesion counts on 59 evaluable patients participating for at least an additional three months were analysed (Table 2). Twenty-six patients were using adapalene from the beginning of the study and 33 patients switched without a wash-out period from tretinoin $0.025 \%$ gel to adapalene $0 \cdot 1 \%$ solution. Thirty-six patients continued the trial for the 12-month duration. It is interesting to note that the patients who started with tretinoin and switched to adapalene showed a slight increase in reduction of total lesions after an additional long-term treatment as compared to the end of a 12 week treatment. The overall reduction of total lesions was similar in these two groups after 12 months of treatment.

\section{Safety}

Discontinuation because of adverse events was reported in 12 patients in the adapalene group, eight of which were judged related or possibly related to the study drug. In the tretinoin group, six patients discontinued, four of which were at least possibly related. The reason for discontinuation in both groups was either acne flare or skin discomfort/dryness/ erythema. A total of 16 patients with adapalene and nine with tretinoin experienced one or several cutaneous adverse events (skin discomfort, erythema, dry skin, pruritus, skin irritation and acne flare). Generally

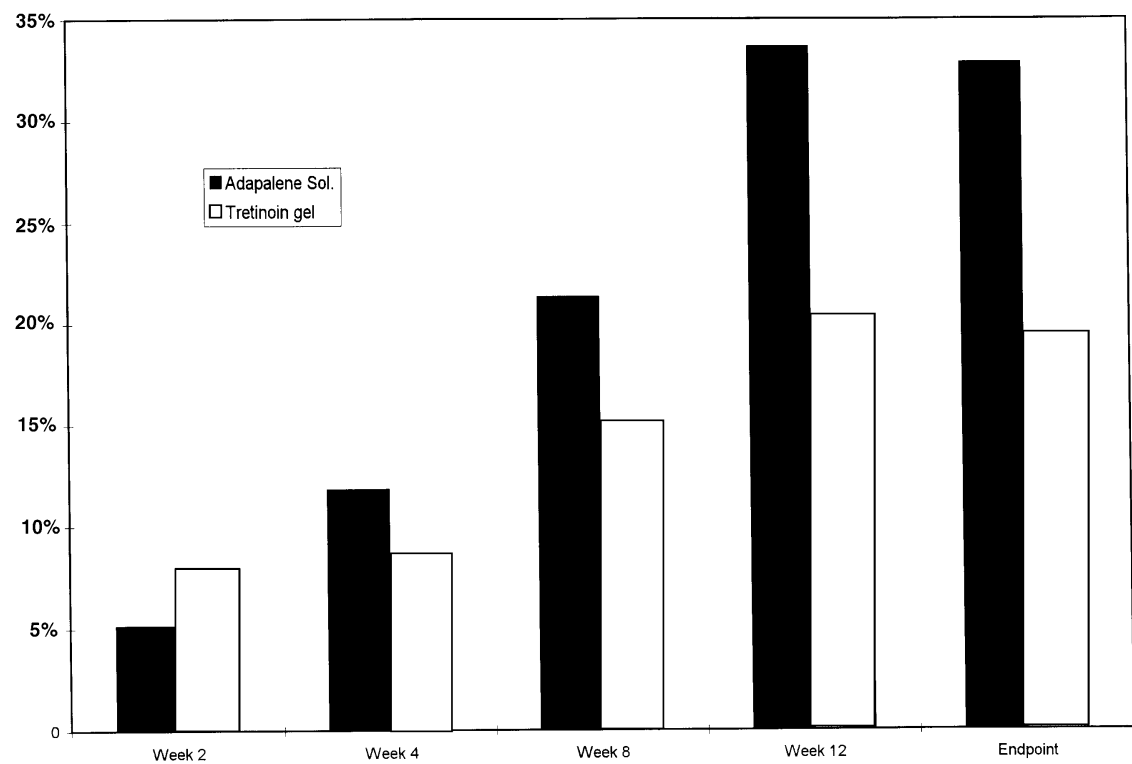

Figure 5. Percentage of patients with $>75 \%$ improvement in open comedone counts

Table 2 Mean lesion count and percentage reduction in total lesions of patients participating in the extension period

\begin{tabular}{|c|c|c|c|c|c|c|c|c|}
\hline \multirow{2}{*}{$\begin{array}{l}\text { Patients originally } \\
\text { assigned to } \\
\text { study treatment }\end{array}$} & \multicolumn{2}{|c|}{ Baseline } & \multicolumn{3}{|c|}{ Week 12 (blinded phase) } & \multicolumn{3}{|c|}{ Month 12 (extension period) ${ }^{*}$} \\
\hline & $n$ & mean & $n$ & mean & $\%$ reduction & $n$ & mean & $\%$ reduction \\
\hline Adapalene & 26 & $76 \cdot 5$ & 26 & $26 \cdot 7$ & 65 & 17 & $25 \cdot 8$ & 66 \\
\hline Tretinoin & 33 & $76 \cdot 5$ & 33 & $34 \cdot 2$ & 55 & 19 & $25 \cdot 9$ & 66 \\
\hline
\end{tabular}

"all patients under adapalene solution 


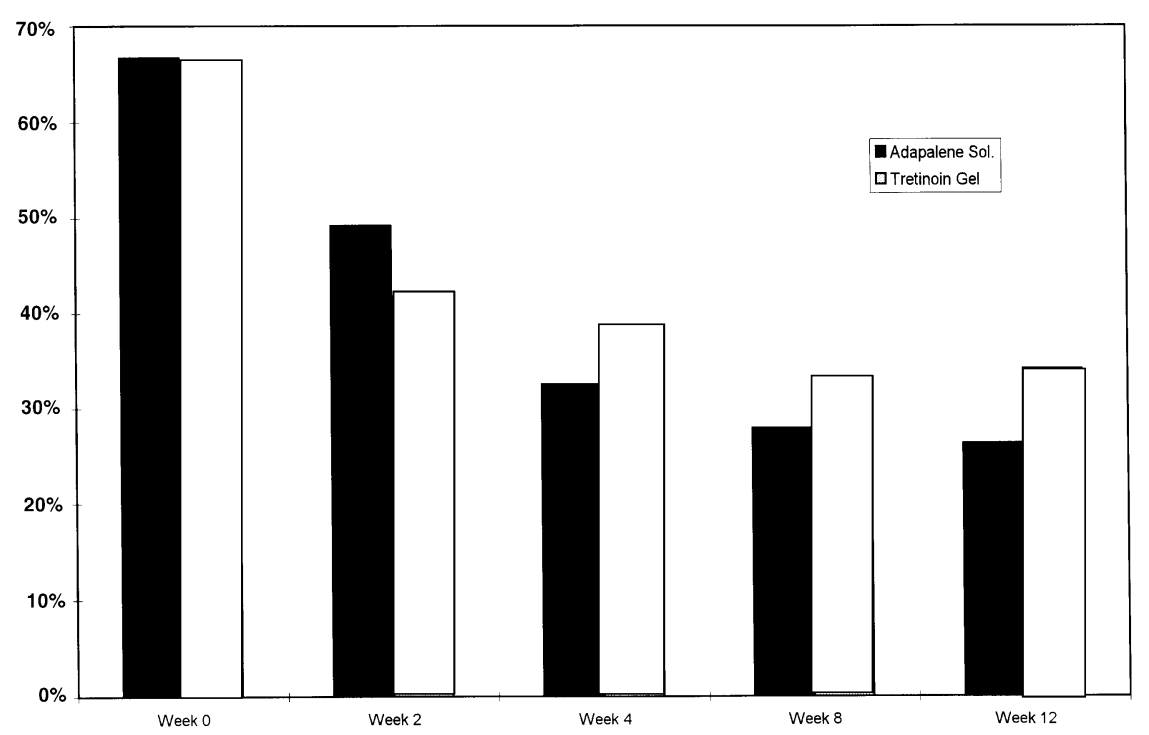

Figure 6. Percentage of patients presenting oiliness moderate skin discomfort, erythema or dry skin appeared during the first days of treatment and resolved with continued or discontinued drug use. Other cutaneous adverse events had a frequency of less than $1 \%$ and were generally mild or moderate.

Concerning signs of cutaneous tolerance, no statistical differences were detected for erythema, dryness or scaling. Scaling increased during the first two weeks of treatment only to decrease steadily in both treatment groups $(52 \%$ with some degree of scaling). As with scaling, the majority of dryness incidents were mild. Sixty-three per cent of the adapalene-treated patients experienced dryness compared with $61 \%$ with tretinoin at week 2 .

Burning and pruritus between and immediately after application were assessed as symptoms of cutaneous tolerability. These symptoms generally increased during the first two weeks of treatment and then subsided slightly during the study. At week 2, there was a total of $45 \%$ of the adapalene treated patients and $55 \%$ of the tretinoin treated patients who described an immediate burning sensation after application. At the end, only 30\% (adapalene) and 34\% (tretinoin) reported the same discomfort. A significant difference was detected at week 4 favouring adapalene (30\% vs. $43 \%$ with tretinoin, $P<0 \cdot 05$ ). Itching, usually mild, was experienced by $38 \%$ of adapalene patients and $20 \%$ of the tretinoin patients during the first two weeks, this difference being statistically significant in favour of tretinoin gel. Immediately after application, itching occurred in 50\% with adapalene solution and $18 \%$ with tretinoin, showing significant differences at all time points in favour of tretinoin.
Oiliness decreased with time from baseline with $66 \%$ of the patients presenting some degree of oiliness (mild to severe) in both groups, to week 12 with $26 \%$ presenting mild to moderately oily skin in the adapalene group and $34 \%$ in the tretinoin group (Fig. 6). No 'severe' scoring was reported after treatment.

\section{Laboratory data}

Biological data involving liver, kidney, haematology, metabolic/electrolyte abnormalities and urinalysis were collected for a total of 74 patients at baseline and week 12 . At week 12 , only 15 patients with adapalene and 15 with tretinoin were considered evaluable for laboratory analysis. No apparent clinically significant abnormal trends were observed in either treatment group; isolated instances of changes from baseline were indicative of normal fluctuations in laboratory data and of non-treatment-related physiological changes.

\section{Discussion}

This multicentre study was conducted to show the efficacy and safety over a 12-week period in acne patients, comparing two topical treatments, adapalene $0 \cdot 1 \%$ solution in a new formulation and tretinoin $0.025 \%$ gel. Thirty-six patients followed the extension period for up to 12 months to assess long-term safety with adapalene solution. In this study, adapalene $0.1 \%$ solution was shown to be clinically and statistically equivalent to tretinoin $0.025 \%$ gel for the treatment of acne. Both treatments were highly effective in treating 

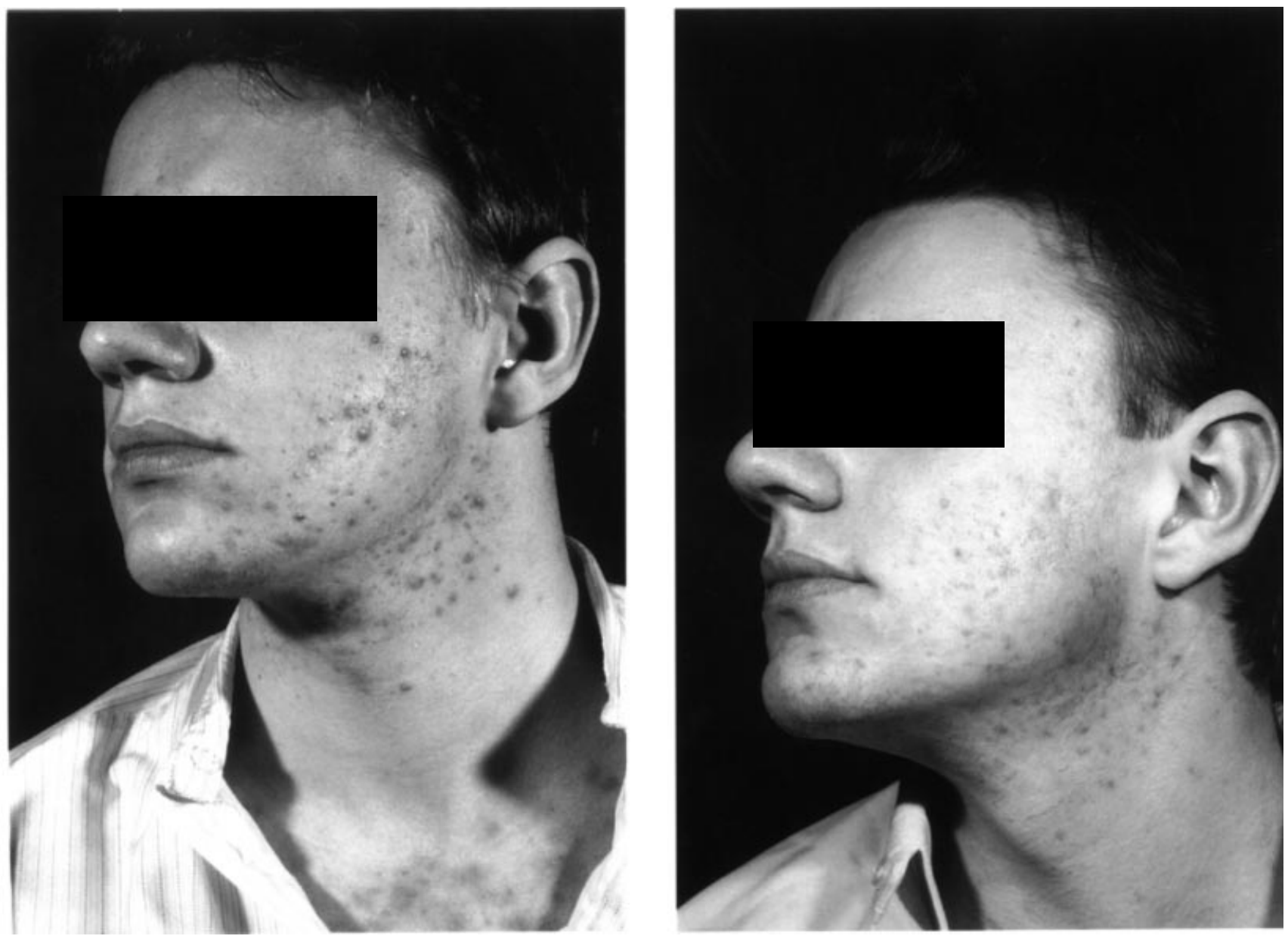

Figure 7. Acne patient treated with adapalene $0 \cdot 1 \%$ solution (a) before treatment; (b) after treatment. Note the absence of apparent irritation.

non-inflammatory and inflammatory lesions. Adapalene $0.1 \%$ solution was somewhat better in the treatment of open comedones. The efficacy rates of both treatments were comparable to those seen in other studies with adapalene. ${ }^{1-3,8}$ This study also supports the findings of previous studies involving other alcoholic preparations. ${ }^{9,10}$

Owing probably to differences in the formulation of the two products, adapalene occasionally produced more skin discomfort due to pruritus. Tretinoin occasionally produced more burning immediately after application at week 4 . All other parameters (erythema, scaling, oiliness, dryness) were comparable. Oiliness decreased from baseline to the end of the study from two thirds of the patients presenting some degree to about one third at week 12 . Both treatments reduced oiliness, adapalene solution numerically, though not significantly, somewhat more than tretinoin. As expected with retinoid-like topical treatments, dryness and scaling are common side-effects, occurring mainly during the first two weeks and subsiding even with the continuation of the treatment. Seventy patients were treated for more than three months and data from 36 patients are available for a treatment period of up to 12 months. Safety-related occurrences did not increase in frequency during the additional nine-month treatment period, even in patients whose treatment was changed from tretinoin to adapalene.

Finding the ideal acne product is a daily clinical challenge for the dermatologist and use of an effective and well-tolerated product is important for patient compliance. As demonstrated in this trial, adapalene solution was both effective and well tolerated. The solution is cosmetically acceptable to an already oily skin surface. The alcoholic solution is easily spread over the face and, in addition, absorbs quickly into the skin allowing the patients to continue their daily routine without interruption. 

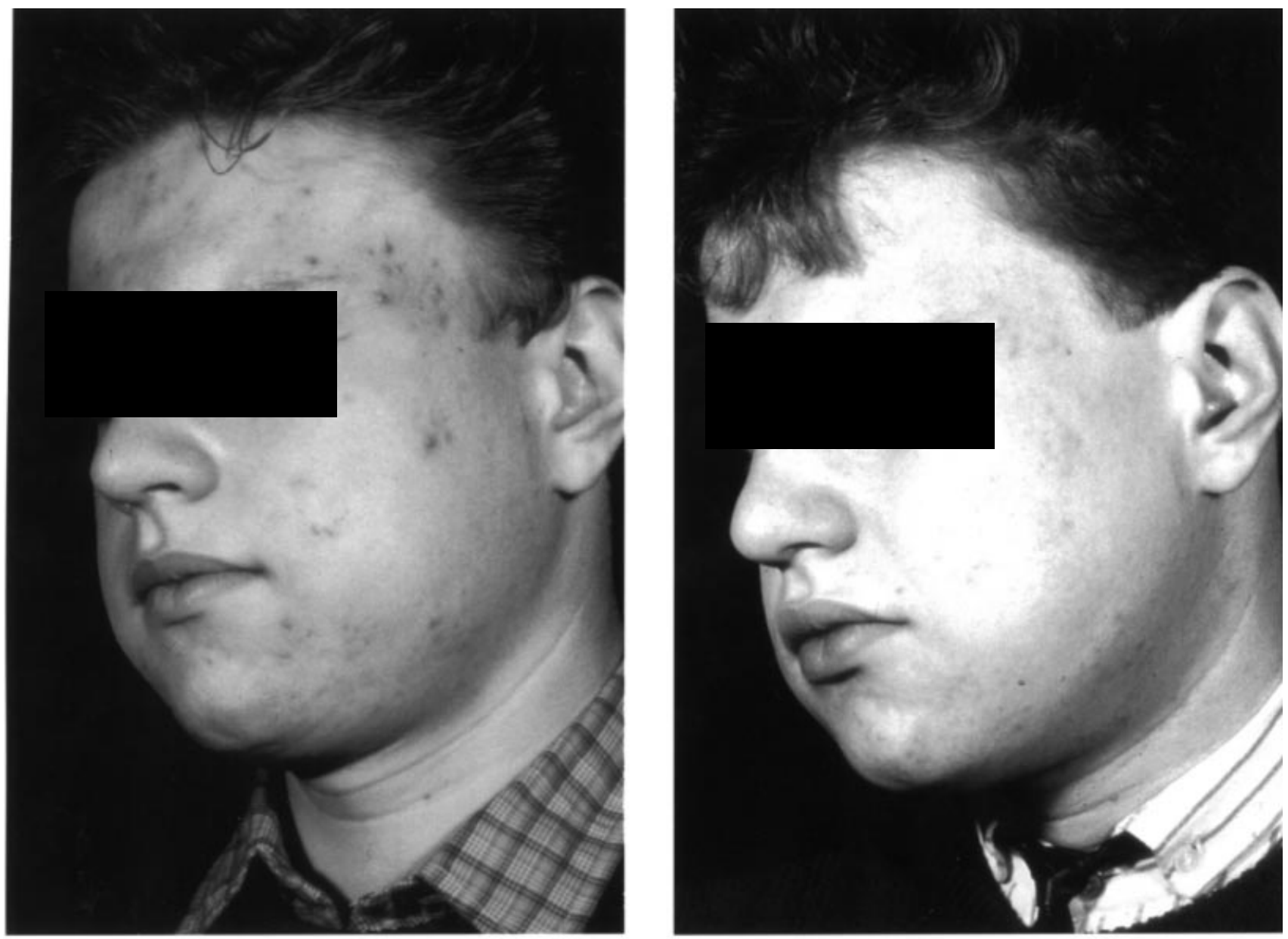

Figure 8 . Acne patient treated with adapalene $0 \cdot 1 \%$ solution (a) before treatment; (b) after treatment. Note the absence of apparent irritation.

In conclusion, adapalene $0 \cdot 1 \%$ solution is comparable to tretinoin $0 \cdot 025 \%$ gel in efficacy and tolerance. The laboratory data showed no abnormal trends with either treatment.

\section{References}

1 Shalita A, Weiss JS, Chalker DK et al. A comparison of the efficacy and safety of adapalene gel $0 \cdot 1 \%$ and tretinoin gel $0.025 \%$ in the treatment of acne vulgaris: a multicenter trial. J Am Acad Dermatol 1996; 34: 482-5.

2 Cunliffe WJ, Caputo R, Dreno B et al. Efficacy and safety comparison of adapalene $(\mathrm{CD} 271)$ gel and tretinoin gel in the topical treatment of acne vulgaris. A European multicenter trial. J Dermatol Treatment 1997; 8: 173-8.

3 Cunliffe WJ, Caputo R, Dreno B. Clinical efficacy and safety comparison of adapalene gel and tretinoin gel in the treatment of acne vulgaris: Europe and U.S. multicenter trials. I Am Acad Dermatol 1996; 36: S126-34.
4 Clucas A, Verschoore M, Sorba V. Adapalene $0 \cdot 1 \%$ gel is better tolerated than tretinoin $0 \cdot 025 \%$ gel in acne patients. J Am Acad Dermatol 1997; 36: S116-18.

5 Shroot B, Michel S. Pharmacology and chemistry of adapalene. J Am Acad Dermatol 1997; 36: S96-103.

6 Allec J, Chatelus A, Wagner N. Skin distribution and pharmaceutical aspects of adapalene gel. J Am Acad Dermatol 1997; 36: S119-25.

7 Burke BM, Cunliffe WJ. The assessment of acne vulgaris -the Leeds technique. Br J Dermatol 1984; 111: 83-92.

8 Alirezai M, Meynadier J, Jablonska S et al. Etude comparative de l'efficacité et de la tolérance de gels d';adapalène à $0 \cdot 1$ et $0 \cdot 03$ p. 100 et d'un gel de trétinoïne à 0.025 p. 100 dans le traitement de l'acné. Ann Dermatol Venereol 1996; 123: $165-70$.

9 Verschoore M, Langner A, Wolska H et al. Efficacy and safety of CD 271 alcoholic gels in the topical treatment of acne. $\mathrm{Br} \mathrm{J}$ Dermatol 1991; 124: 368-71.

10 Verschoore M, Langner A, Wolska $\mathrm{H}$ et al. Vehicle controlled study of CD 271 lotion in the topical treatment of acne vulgaris. J Invest Dermatol 1993; 100: 221. 